\title{
Temperature as a Single On-Off Parameter Controlling Nanoparticles Growing, Stabilization and Fast Disentanglement
}

\author{
By Paula M. López-Pérez, Ricardo M. P. da Silva,* Iva Pashkuleva, Julio San Román,* \\ and Rui L Reis
}

The small size of nanoparticles, together with increased surface area and reactivity, allows them to translocate cell membranes, target specific tissues or organs (by adsorption or surface binding of signaling molecules), and catalyze chemical reactions. ${ }^{[1]}$ Owing to its unique properties, organic nanoparticles (polymer or lipid) hold a great potential to be used in various biomedical applications, especially as intracellular drug and gene delivery systems. ${ }^{[2,3]}$ Small size is a key attribute enabling nanoparticles to overcome various biological barriers such as the blood-brain barrier, the reticuloendothelial system (RES), tumor blood vessels with enhanced permeability, extracellular matrix components, cell membrane and other intracellular barriers. ${ }^{[1]}$ Herein we describe a method for the synthesis of thermoresponsive nanoparticles that provides a good control over particle size (between 35-200 $\mathrm{nm}$ ). We have designed random thermoresponsive terpolymers that possess a fine balance between hydrophobic attractive forces and electrostatic repulsion above the lower critical solution temperature (LCST). In the literature, block copolymers have been a primary choice to trigger macromolecular assembly in aqueous environment, based on a lipidlike amphiphilic nature. ${ }^{[4-7]}$ In this work, the choice of random copolymers instead was inspired by their resemblance with the intercalated nature of hydrophobic and charged residues found in the primary structure of proteins. The method reported herein allows nanoparticles to be processed in aqueous medium without surfactants, using temperature as a single on-off parameter controlling particle growing, stabilization and fast disentanglement.

The most widespread methods for nanoparticles production include the previous preparation of an emulsified system. ${ }^{[8]}$

[*] Dr. P. M. López-Pérez, Dr. R. M. P. da Silva, Dr. I. Pashkuleva, Prof. R. L. Reis

3B's Research Group - Biomaterials, Biodegradables, and Biomimetics

University of Minho Headquarters of the European Institute of Excellence on Tissue Engineering and Regenerative Medicine AvePark, 4806-909 Taipas, Guimarães, (Portugal)

IBB - Institute for Biotechnology and Bioengineering

PT Government Associated Laboratory

Braga (Portugal)

E-mail: ricardosilva@dep.uminho.pt

Prof. J. San Román

Institute of Polymer Science and Technology

CSIC., C/Juan de la Cierva, 3, 28006-Madrid (Spain)

CIBER-BBN (Spain)

E-mail: jsroman@ictp.csic.es

DOI: 10.1002/adma.201001717
Thereafter, particles are formed in the discontinuous phase either by polymer precipitation/gelation or by monomer polymerization. Two immiscible phases are required and the oil phase is not always easy to remove. Surfactants are often required to stabilize the emulsion and to avoid nanoparticle aggregation. Furthermore, emulsion-based methods often require use of crosslinkers. ${ }^{[8]}$

Alternative methods to prepare nanoparticles in aqueous medium are based on polymer precipitation or macromolecular self-assembly in conditions of spontaneous dispersion formation. These methods avoid the use of organic solvents, crosslinkers and/or surfactants. ${ }^{[8]}$ Thermoresponsive polymers offer unique properties that have been elegantly adapted to trigger the spontaneous formation of nanoparticles, which can be used for burst drug release on demand. ${ }^{[2,6,7]}$

Thermoresponsive polymers such as poly( $N$-isopropylacrylamide) (PNIPAAm) are hydrophilic at low temperature and became hydrophobic above the LCST. Graft or linear block copolymers using PNIPAAm can form core-shell micellar assemblies, with the thermoresponsive building block working as either the solvated shell ${ }^{[4,5]}$ or the hydrophobic core, ${ }^{[6,7]}$ depending on the nature of the other block and on the temperature. If PNIPAAm is coupled to more hydrophobic blocks, stable polymeric micelles are formed below the LCST. ${ }^{[4,5]}$ Hydrophobic drugs can be loaded into the inner corona, while the hydrophilic PNIPAAm shell permits the aqueous solubilization and temperature-responsiveness. This architecture does not differ from other non-thermoresponsive micelles assembled from block copolymer amphiphiles, except in that the hydrophilic shell can turn hydrophobic upon temperature increase, ${ }^{[4,5]}$ eventually destabilizing the inner core of the micelle and triggering drug release. ${ }^{[4]}$ The core-shell structure is formed when the polymer, previously dissolved in a water miscible organic solvent, is mixed with water. ${ }^{[4,5]}$

In the case of block copolymers of PNIPAAm with a hydrophilic block such as poly(ethylene glycol) $(\mathrm{PEG})^{[6]}$ or single-stranded DNA, ${ }^{[7]}$ the macromolecule can be dissolved in water below the LCST. Stable core-shell nanoparticles are produced above the LCST, when the PNIPAAm block became hydrophobic. The hydrophilic block forms a hydrated shell that prevents further aggregation of collapsed PNIPAAm chains. ${ }^{[6,7]}$ Inducing macromolecular assembly above the LCST in water circumvents the need of using organic solvents. Furthermore, these methods allow nanoparticles to be reversibly disassembled lowering the temperature, which might be useful to release drugs locally applying simple ice packs or clinical instruments such as deeply penetrating cryoprobes. ${ }^{[9]}$ 
Similarly, polymeric nanogels can also be obtained with thermoresponsive random copolymers, being macromolecular assembly in water induced above the LCST. In this case, the electrostatic repulsion between charged surfaces stabilizes the colloidal nanoparticles above the LCST. ${ }^{[10,11]}$ In a recent work, ${ }^{[11]}$ we have found that charged terpolymers composed of NIPAAm, $N$-tert-butylacrylamide (NTBAAm) and 2-Acrylamido2-methyl-1-propanesulphonic acid (AMPS) form metastable colloidal nanoparticles upon heating above the LCST. The original method described herein is based on the distinct aggregationredissolution behavior observed for some of these thermoresponsive terpolymers.

Linear random terpolymers were synthesized by radical polymerization containing a constant ratio of $5 \%$ of AMPS in feed. The small ratio of charged monomer ensures enough electrostatic repulsion intended for nanoparticles stabilization, but is not enough to disrupt the discontinuous type coil-toglobule transition. ${ }^{[12]}$ Terpolymer hydrophobicity was varied by changing the ratio between NIPAAm and a more hydrophobic monomer (NTBAAm) in order to adjust the LCST. Terpolymers with NIPAAm/NTBAAm/AMPS ratios of $80 / 15 / 5$ and $60 / 35 / 5$ were studied, representing two different aggregation-redissolution behaviors, which changed above a critical amount of NTBAAm (0.25-0.30). ${ }^{[1]}$

Turbidimetry measurements were performed to picture the macroscopic observation of the phase separation (Figures 1a and $1 \mathrm{~b}$, right $\gamma$-axis). The cloud point temperature (CPT) was extracted from heating scans at the transmittance decrease onset, whereas a redissolution temperature can be observed on cooling. Dynamic light scattering (DLS) provides an insight of the early macromolecular aggregation (Figures $1 \mathrm{a}$ and $1 \mathrm{~b}$, left $\gamma$-axis). Above the LCST a sharp increase of the hydrodynamic diameter $\left(D_{\mathrm{h}}\right)$ is observed indicating the aggregation of individual ionomer chains in solution. Although the size of the polymers synthesized by us did not allow direct observation of the molecular contraction during coil-to-globule transition (single molecules are not observed above the LCST), this first aggregation step provides a good estimation of the LCST. ${ }^{[13]}$ For the sake of clarity, we will use the term LCST to denominate this early aggregation observed by DLS and the CPT to denote the value obtained by turbidimetry.

The more hydrophobic polymer $(60 / 35 / 5)$ presented lower LCST, CPT and redissolution temperature. The redissolution temperature was lower than the CPT for both copolymers (Figures $1 \mathrm{a}$ and $1 \mathrm{~b}$ ). The general aggregation profile observed by DLS was also similar for both copolymers (Figures 1a and 1b). An early aggregation (LCST) occurs with formation of small aggregates that remain stable at temperatures near and above the LCST. Further increasing the temperature, a "massive" coagulation happens with the formation of bigger aggregates. This "massive" coagulation corresponds to the CPT observed by turbidimetry.

The copolymer containing lower NTBAAm ratio (80/15/5) (Figure 1a) showed a sharp macroscopic phase separation on heating and a fast increase of the $D_{\mathrm{h}}$ in the second aggregation step. Moreover, it was observed that redissolution on cooling occurs at higher temperatures than the early aggregation observed by DLS. It seems that the colloidal particles formed on heating are disrupted on cooling before reaching the LCST.
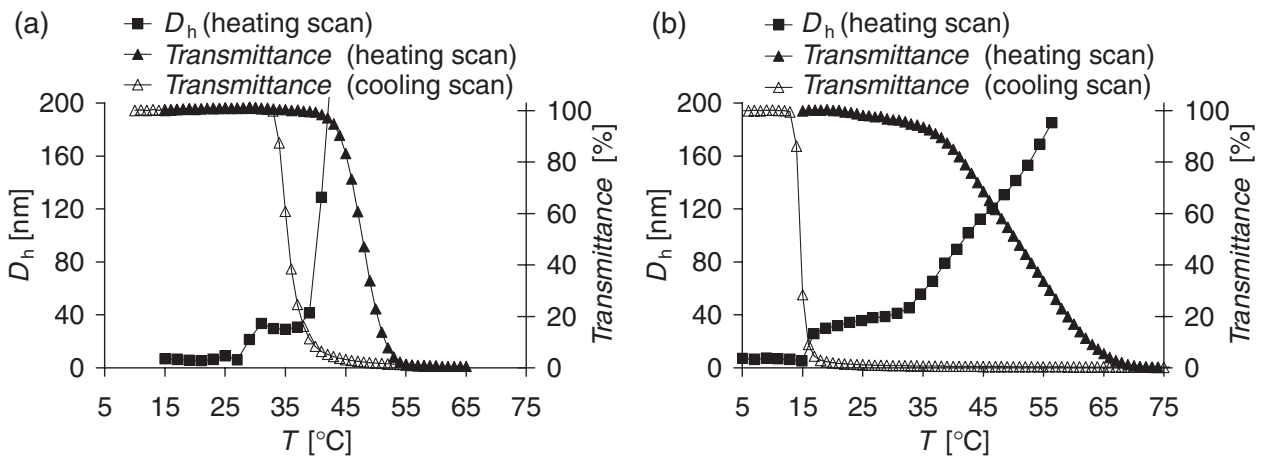

(c)

(d)
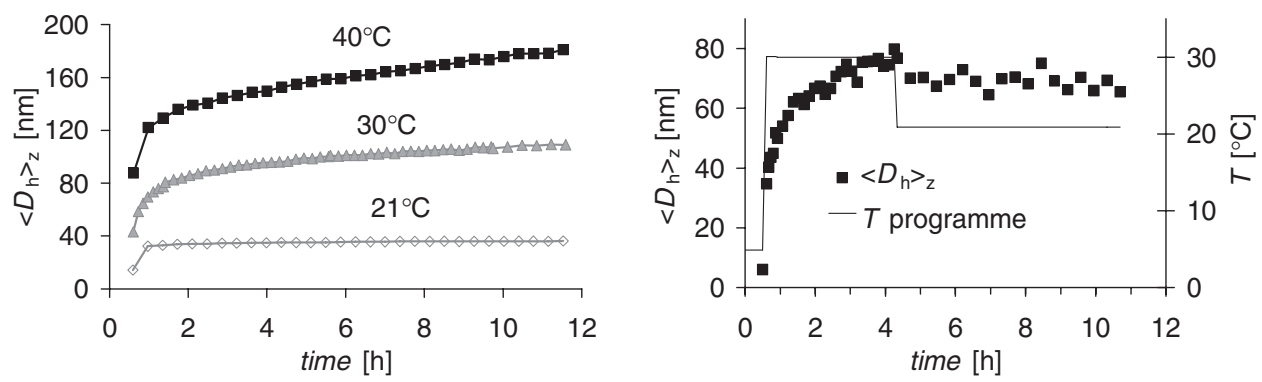

Figure 1. Hydrodynamic diameter $\left(D_{\mathrm{h}}\right)$ and solution turbidity (Transmittance) changes observed as a function of temperature for (a) $80 / 15 / 5$ and (b) 60/35/5. (c) Aggregation isotherms for 60/35/5 above the LCST. (d) Nanoparticles size control using a temperature program. All measurements were done at polymer concentration of $1 \mathrm{~g} \mathrm{~L}^{-1}$ in $\mathrm{NaCl}$ aqueous solution $(0.120 \mathrm{M})$. 
On the other hand, the more hydrophobic copolymer $(60 / 35 / 5)$ showed slower macroscopic phase separation (Figure $1 \mathrm{~b}$ ). This is in agreement with the slower second aggregation observed by DLS at higher temperature (Figure 1b). In this case, the large aggregates formed during the heating scan seem to dissolve only after cooling below the LCST (Figure 1b), at temperature lower than the early small metastable nanoaggregates formed on heating.

Aggregation isotherms were determined for 60/35/5 (Figure 1c). Samples were initially equilibrated at $5{ }^{\circ} \mathrm{C}$ in order to assure complete dissolution. Thereafter, solutions were submitted to a temperature jump and z-average $D_{\mathrm{h}}\left(\left\langle D_{\mathrm{h}}\right\rangle_{\mathrm{z}}\right)$ was recorded at constant temperature. It was observed that metastable colloidal particles of around $35 \mathrm{~nm}$ were formed at $21^{\circ} \mathrm{C}$; size remained stable for at least $12 \mathrm{~h}$. When the solution was kept at $30^{\circ} \mathrm{C}$ the $\left\langle D_{\mathrm{h}}\right\rangle_{\mathrm{z}}$ increased with the time indicating that aggregation occurs slowly at this temperature. The aggregation rate increases with the temperature as could be verified by measuring the aggregation isotherm at $40{ }^{\circ} \mathrm{C}$ (Figure 1c). Taking into consideration the smooth growth of colloidal particles observed at 30 and $40{ }^{\circ} \mathrm{C}$ (Figure 1c), together with the fact that larger colloidal aggregates only completely redissolve when cooled below the LCST (Figure 1b), we hypothesized that the aggregates, meanwhile formed at higher temperatures, would not disentangle and could be stabilized at temperatures near and above the LCST $\left(21^{\circ} \mathrm{C}\right)$. In fact, it can be observed in Figure $1 \mathrm{~d}$ particles that have been grown at $30^{\circ} \mathrm{C}$ for some time. When temperature is decreased to $21{ }^{\circ} \mathrm{C}$ the $\left\langle D_{\mathrm{h}}\right\rangle_{\mathrm{z}}$ remains constant. In a separate experiment, we observed that nanoparticle size was constant for at least one week (not shown). This behavior can be used to deliver customized particle size, just maintaining the polymer solution at high temperature for some time and decreasing the temperature to $21^{\circ} \mathrm{C}$ when particles achieve the desired size. Figures $2 \mathrm{a}$ and $2 \mathrm{~b}$ show the hydrodynamic size of nanoparticles obtained at 30 and $40{ }^{\circ} \mathrm{C}$, respectively. The polydispersity determined from the particle size distribution was found to be in the range $0.125-0.15$, as shown in Figure 2c for the nanoparticles obtained at $30^{\circ} \mathrm{C}$. Typical bell-shaped monomodal size distributions were obtained for particles stabilized at $21{ }^{\circ} \mathrm{C}$, irrespectively the temperature used in the growing stage (see Figure $2 \mathrm{~d}$ ). A schematic representation of the manipulation of nanoparticles size is depicted in Figure 2e. Nanoparticles with sizes between 40 and $100 \mathrm{~nm}$ can be obtained by growing them at $30^{\circ} \mathrm{C}$ for different time periods (Figure $2 \mathrm{a}$ ). When colloidal nanoparticles were formed at $40^{\circ} \mathrm{C}$, sizes in the range $100-200 \mathrm{~nm}$ were obtained (Figure 2b) Nanoparticles can also be reversibly disentangled at temperature below the LCST, with recovery of soluble terpolymer chains. This type of systems might be very useful for instance in intracellular drug delivery and actuators for burst release applications.

Electrostatic stabilization through the anionic groups of AMPS was initially though to be the most important stability mechanism for this system. Therefore, electrophoretic mobility $\left(\mu_{\mathrm{e}}\right)$ measurements were performed to evaluate particles

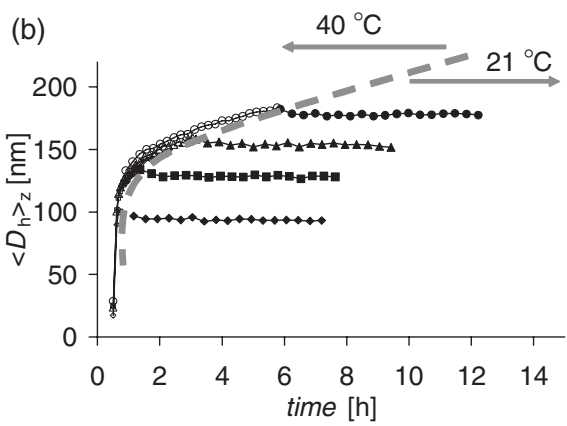

(e)
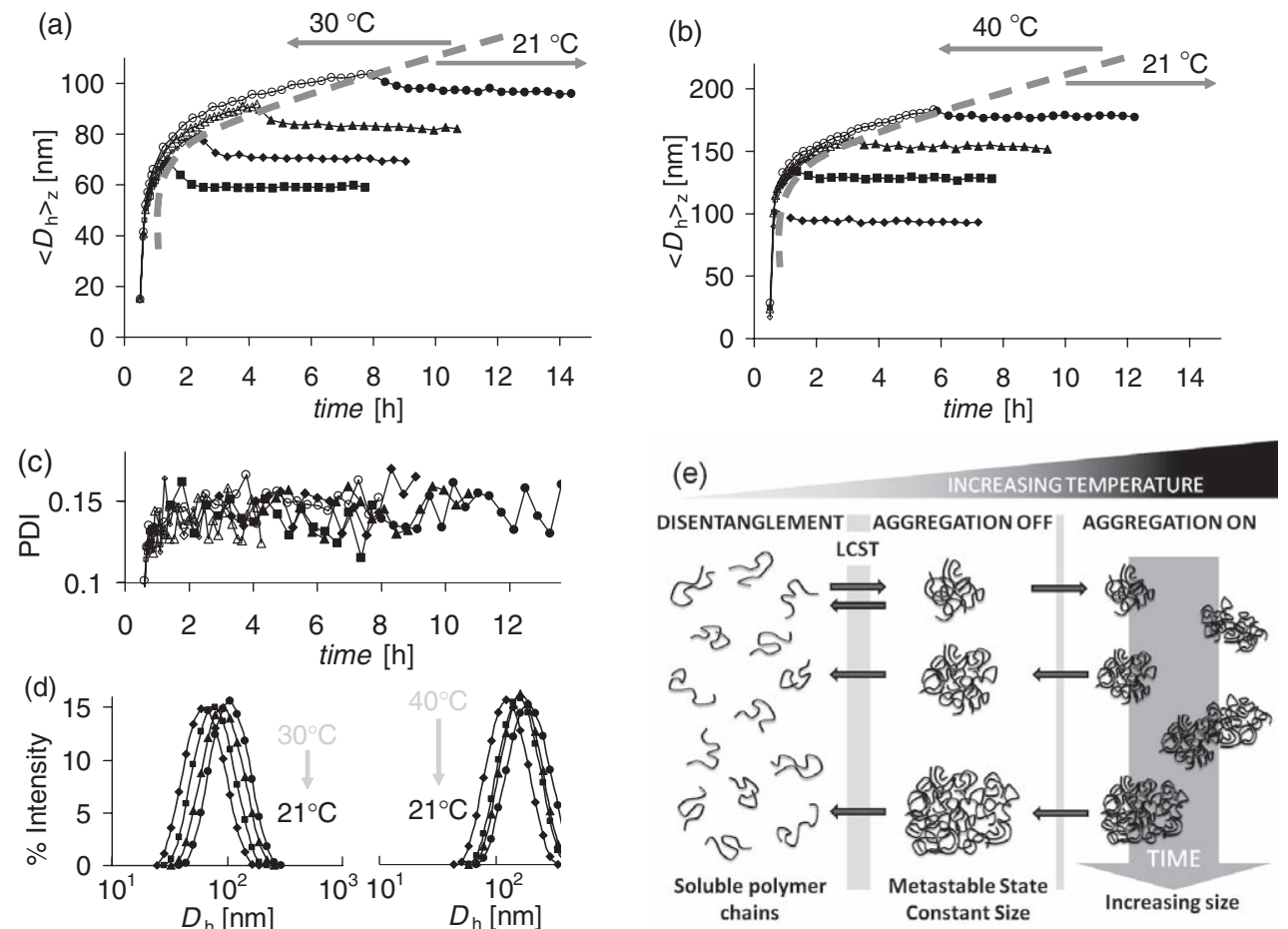

Figure 2. Nanoparticles z-average hydrodynamic diameter $\left(\left\langle D_{h}\right\rangle_{z}\right)$ observed as a function of temperature for $60 / 35 / 5$. Aggregation is triggered at (a) $30^{\circ} \mathrm{C}$ or at (b) $40^{\circ} \mathrm{C}$ for well-defined time periods and subsequently inhibited at $21^{\circ} \mathrm{C}$. (c) Polydispersity (PDI) of particles obtained at $30^{\circ} \mathrm{C}$ and (d) typical size distributions of particles measured at $21^{\circ} \mathrm{C}$ after a growing stage at 30 or $40^{\circ} \mathrm{C}$. All measurements were done at polymer concentration of $1 \mathrm{~g} \mathrm{~L}^{-1}$ in $\mathrm{NaCl}$ aqueous solution $(0.120 \mathrm{M})$. (e) Schematic representation of the nanoparticles size manipulation using the thermoresponsive aggregation behavior. 
(a)

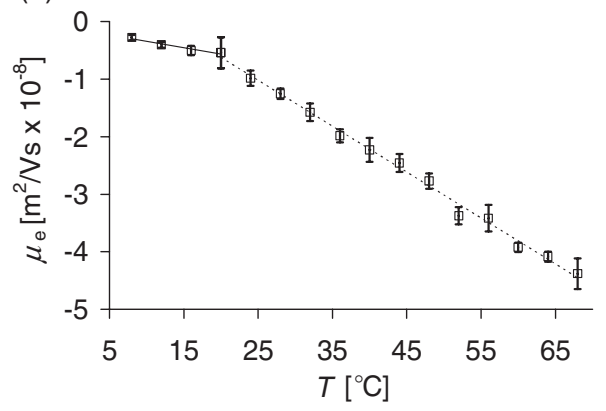

(b)

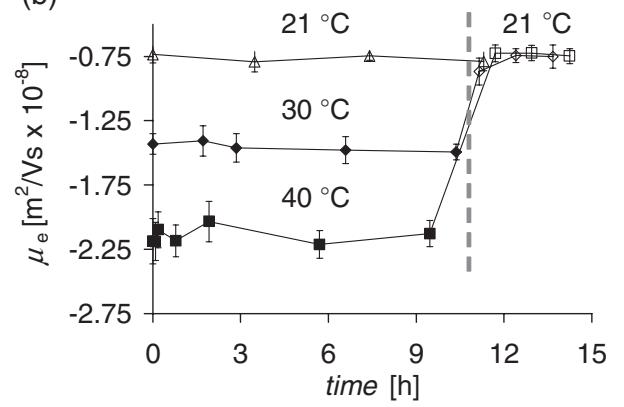

Figure 3. (a) Electrophoretic mobility $\left(\mu_{\mathrm{e}}\right)$ observed as a function of temperature for $60 / 35 / 5$. Linear regression trend lines are shown independently for the data sets above and below $21^{\circ} \mathrm{C}$. (b) $\mu_{\mathrm{e}}$ measured isothermally for the studied aggregation kinetic stages, followed by isothermal determination at $21{ }^{\circ} \mathrm{C}$. All measurements were done at polymer concentration of $1 \mathrm{~g} \mathrm{~L}^{-1}$ in $\mathrm{NaCl}$ aqueous solution $(0.120 \mathrm{M})$.

surface charge. It was observed (Figure 3a) that below the LCST $\mu_{\mathrm{e}}$ value remains sensibly constant. Above the LCST the absolute value of $\mu_{\mathrm{e}}$ increases in a pseudo-linear manner with the temperature. In general, a higher $\left|\mu_{\mathrm{e}}\right|$ would imply an improved stability because of the electrostatic repulsion. However we observed exactly the opposite. Interestingly, $\mu_{\mathrm{e}}$ was independent on both temperature scanning rate and particle size. As seen in Figure $3 \mathrm{~b}$ the electrophoretic mobility at a fixed temperature remained constant during at least $9 \mathrm{~h}$, regardless occurrence or not of colloidal aggregation. It was also observed that decreasing the temperature to $21{ }^{\circ} \mathrm{C}$ after this time period at 30 or $40{ }^{\circ} \mathrm{C}$ the $\mu_{\mathrm{e}}$ recovers the value initially obtained at $21^{\circ} \mathrm{C}$. The straightforward correlation between $\mu_{\mathrm{e}}$ and temperature seems to indicate that the on-off aggregation above the LCST is ruled by surface phenomenon. It is well known that the driving force of the aggregation in thermoresponsive polymers is an increased hydrophobicity above the LCST. ${ }^{[14]}$ Similarly, the driving force for increasing $\left|\mu_{\mathrm{e}}\right|$ with temperature should also be an increasing hydrophobicity. Thus, if aggregation happens at higher surface charge, it is also because hydrophobic forces have been enhanced, being at a certain point able to counteract the electrostatic repulsion. In order to explain why aggregation is much faster for $\mu_{\mathrm{e}}$ values where typically colloidal stability is attained, we might speculate that the non-crosslinked nature of the polymer aggregates permits molecular rearrangements that minimize surface charge electrostatic repulsion when different colloidal particles approach each other. On the other hand, the low $\left|\mu_{\mathrm{e}}\right|$ values also did not completely justify the colloidal stability found for temperatures closer to the LCST $\left(21^{\circ} \mathrm{C}\right)$. Consequently, a different mechanism should be actuating, providing an additional (or main) energy barrier. A possible explanation can be an increased steric repulsion provided by an increased mobility of polymeric chains (lower polymer surface density) at lower temperature. In fact, the lower $\left|\mu_{\mathrm{e}}\right|$ values are consistent with the hypothesis of lower polymer surface density.

In summary, we propose herein an original method to produce thermoresponsive nanoparticles by means of a temperature cycle. This method has several advantages; the nanoassemblies are formed in aqueous medium and surfactants are not needed for particles stabilization. The slow aggregation observed above the LCST was used to obtain a fine control over the particle size. Nanoparticles, grown at a higher temperature
(30 and $40{ }^{\circ} \mathrm{C}$ ), are stabilized at the desired size by decreasing the temperature to $21{ }^{\circ} \mathrm{C}$. Finally, complete disentanglement of the nanoparticles can be triggered by further decreasing the temperature (below $16{ }^{\circ} \mathrm{C}$ ). The thermoresponsive system reported here may be potentially useful for a range of applications, including drug and gene delivery, biosensing, or separation and purification of biological molecules and cells. Moreover, we believe that it would be possible to extend the method to other thermoresponsive polymers, both cationic and anionic, by fine tuning the balance between electrostatic repulsion and polymer hydrophobicity.

\section{Experimental Section}

$\mathrm{N}$-isopropylacrylamide (NIPAAm, Acros Organics) and 2,2'-Azobisisobutyronitrile (AIBN) (Fluka) were recrystallized from $n$-hexane/ diethyl ether (5:1) and methanol, respectively. 2-Acrylamido-2-methyl-1. propanesulphonic acid (AMPS) and N-tert-butylacrylamide (NTBAAm) (Sigma-Aldrich) were used as received.

Linear random terpolymers P(NIPAAm-co-NTBAAm-co-AMPS) were synthesized as previously described. ${ }^{[1]}$ Briefly, monomers (total concentration $0.5 \mathrm{M}$ ) were dissolved in isopropanol/water (50:50) and AIBN (1 mol\% of total monomers) was added. Two different copolymers were synthesized using feed molar percentages of NIPAAm/NTBAAm/ AMPS in the reaction mixture of $60 / 35 / 5$ and $80 / 15 / 5$, respectively. After deoxygenation with $\mathrm{N}_{2}$ (15 min), the reaction vessel was sealed and kept at $60{ }^{\circ} \mathrm{C}$ for $16 \mathrm{~h}$. Then, polymer solutions were neutralized with $\mathrm{NaOH}$, dialyzed against distilled water (cut-off $3500 \mathrm{Da}$ ) and freezedried. Terpolymers composition was very similar to the reaction feed composition, as determined by Elemental analysis (Leco CHNS-932) and ${ }^{1} \mathrm{H}$ NMR $\left(300 \mathrm{MHz}, \mathrm{CDCl}_{3}\right.$ ) (Varian Inova 300). ${ }^{[1]}$ Molecular weight $(\sim 18 \mathrm{kDa})$ and polydispersity $(\sim 2.7)^{[1]}$ were determined by gel permeation chromatography (GPC) using $\mathrm{LiBr}(0.1 \% \mathrm{w} / \mathrm{v})$ in DMF as eluent at a flow

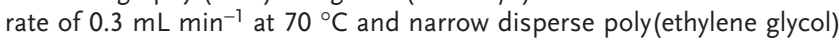
(PEG) as calibration standards.

The cloud point temperature (CPT) of the polymer solutions was measured in a Varian-Cary 3 UV/Visible spectrophotometer, equipped with a Peltier temperature controller. The transmittance $(400 \mathrm{~nm})$ was monitored as a function of temperature under magnetic stirring. Solutions were frozen at $-20{ }^{\circ} \mathrm{C}$ to ensure complete dissolution. Immediately after melting, solutions were placed in a cuvette and heating scans were performed between $15-80{ }^{\circ} \mathrm{C}$ at a scanning rate of $1{ }^{\circ} \mathrm{C} \mathrm{min}^{-1}$. The initially clear polymer solution was used as blank. Cooling scans were performed between $80-5{ }^{\circ} \mathrm{C}$ immediately after heating at the same rate. 
The hydrodynamic diameter was determined by Dynamic Light Scattering (DLS) using a Zetasizer NanoZS Instrument (ZEN3600, Malvern Instruments, Worcestershire, UK) equipped with a $4 \mathrm{~mW}$ He-Ne laser $\left(\lambda_{0}=633 \mathrm{~nm}\right)$ and with non-invasive backscattering (NIBS) detection at a scattering angle of $173^{\circ}$. Both measuring position and attenuator were adjusted automatically before each measurement. Measurements at variable temperature were performed after an equilibration time of at least 2 min.

The electrophoretic mobility was obtained by Laser Doppler Velocimetry (LDV) using a Zetasizer NanoZS Instrument (ZEN3600, Malvern Instruments, Worcestershire, UK) at a scattering angle of $17^{\circ}$ and capillary folded cells (DTS1060, Malvern, Worcestershire, UK). The measurements were performed with an applied voltage of $\pm 20 \mathrm{~V}$ in the temperature range $8-64{ }^{\circ} \mathrm{C}$ after an equilibration time of $4 \mathrm{~min}$. For both DLS and electrophoretic mobility measurements, the terpolymer saline solutions were prepared with ultrapure water and filtered using a $0.20 \mu \mathrm{m}$ disposable PES membrane filter (TPP, Trasadingen, Switzerland).

\section{Acknowledgements}

The authors acknowledge funding from EU Marie Curie Actions, Alea Jacta Est (MEST-CT-2004-008104) and Portuguese Foundation for Science and Technology (FCT) (SFRH/BPD/34545/2007). This work was carried out under the scope of the European NoE EXPERTISSUES (NMP3-CT-2004-500283).

Received: May 10, 2010

Published online: July 22, 2010

[1] a) S. M. Hussain, L. K. Braydich-Stolle, A. M. Schrand, R. C. Murdock, K. O. Yu, D. M. Mattie, J. J. Schlager, Adv. Mater. 2009, 21, 1549; b) M. De, P. S. Ghosh, V. M. Rotello, Adv. Mater. 2008, 20, 4225 .
[2] S. Ganta, H. Devalapally, A. Shahiwala, M. Amiji, J. Control. Release 2008, 126, 187.

[3] a) C. J. F. Rijcken, O. Soga, W. E. Hennink, C. F. v. Nostrum, J. Control. Release 2007, 120, 131; b) J. M. Oliveira, N. Kotobuki, A. P. Marques, R. P. Pirraco, J. Benesch, M. Hirose, S. A. Costa, J. F. Mano, H. Ohgushi, R. L. Reis, Adv. Funct. Mater. 2008, 18, 1840 .

[4] J. E. Chung, M. Yokoyama, T. Okano, J. Control. Release 2000, 65, 93.

[5] a) F. Kohori, K. Sakai, T. Aoyagi, M. Yokoyama, M. Yamato, Y. Sakurai, T. Okano, Colloid Surf. B-Biointerfaces 1999, 16, 195; b) L. Zhang, R. Guo, M. Yang, X. Jiang, B. Liu, Adv. Mater. 2007, 19, 2988.

[6] a) D. Neradovic, O. Soga, C. F. Van Nostrum, W. E. Hennink, Biomaterials 2004, 25, 2409; b) P. W. Zhu, D. H. Napper, Langmuir 2000, 16, 8543; c) M. D. C. Topp, P. J. Dijkstra, H. Talsma, J. Feijen, Macromolecules 1997, 30, 8518; d) X. P. Qiu, C. Wu, Macromolecules 1997, 30, 7921.

[7] T. Mori, M. Maeda, Langmuir 2004, 20, 313.

[8] C. Vauthier, K. Bouchemal, Pharm. Res. 2009, 26, 1025.

[9] S. Qin, Y. Geng, D. E. Discher, S. Yang, Adv. Mater. 2006, 18, 2905.

[10] a) X. Qiu, M. Li, C. M. S. Kwan, C. Wu, J. Polym. Sci. B: Pol. Phys. 1998, 36, 1501; b) X. P. Qiu, C. M. S. Kwan, C. Wu, Macromolecules 1997, 30, 6090.

[11] P. M. Lopez-Perez, R. M. P. da Silva, I. Pashkuleva, F. Parra, R. L. Reis, J. San Roman, Langmuir 2010, 26, 5934.

[12] S. Varghese, A. K. Lele, R. A. Mashelkar, J. Chem. Phys. 2000, 112, 3063.

[13] X. P. Qiu, M. Li, C. M. S. Kwan, C. Wu, J. Polym. Sci. B: Polym. Phys. 1998, 36, 1501.

[14] Y. G. Takei, T. Aoki, K. Sanui, N. Ogata, Y. Sakurai, T. Okano, Macromolecules 1994, 27, 6163. 